\title{
Metodología ecléctica y mediación intercultural en la enseñanza de español a sinohablantes de nivel inicial
}

\author{
Eclectic Methodology and Intercultural Mediation \\ in Teaching Initial Spanish to Native Chinese Speakers
}

\author{
Bega García Viudez \\ Department of Applied Lingüistics and Education Nebrija University, \\ Pirineos 55, 28040 Madrid, Spain \\ e-mail: bgarcia@nebrija.es \\ Carmen Hernández Alcaide \\ Department of Applied Lingüistics and Education, Nebrija University, \\ Pirineos 55, 28040 Madrid, Spain \\ e-mail: chernan@nebrija.es \\ Susana Martín Leralta \\ Department of Applied Lingüistics and Education, Nebrija University, \\ Pirineos 55, 28040 Madrid, Spain \\ e-mail: smartin@nebrija.es
}

\begin{abstract}
The aim of this study is to know the appraisal of the agents involved in the implementation of a course designed for developing the oral communication effectiveness of Chinese workers. For the course, we advocated an eclectic methodology in which teaching resources of different kinds were applied to optimize language learning considering the specific needs of the learners. We decided the participation of two teachers per group and a linguistic and cultural Chinese mediator and we also took into account the methodology currently followed in the Chinese centers: the traditional approach (grammar-translation) and structural (Yang, 2013). In this observational descriptive study, the course participants (6 teachers, 6 assistants and 59 students) answered a questionnaire about different factors such as methodology, design of materials, resources used, use of the mother tongue, learners reactions to certain tasks, role of
\end{abstract}


teachers and assistants. The results show satisfaction with the methodology, resources and materials used.

Keywords: Spanish for Chinese migrants, eclectic methodology, cultural mediation, Spanish as a foreign language

\section{INTRODUCCIÓN}

Esta investigación surge con el fin de facilitar a trabajadores sinohablantes el aprendizaje de la lengua y desarrollar su eficacia comunicativa oral en español, ya que este colectivo presenta unas dificultades de integración específicas, derivadas fundamentalmente de su distancia lingüística y cultural respecto a la sociedad española de acogida.

El proyecto Comunicate en español, dependiente de la Fundación Antonio de Nebrija, está subvencionado por la Consejería de Asuntos Sociales de la Comunidad de Madrid y fue llevado a cabo entre julio de 2014 y julio de 2015 por el Grupo de investigación LAELE (Lingüística aplicada a la enseñanza de lengua extranjera), de la Universidad Nebrija. Dicho proyecto consistía en el diseño de un curso dirigido a desarrollar la eficacia comunicativa oral de trabajadores chinos. Para ello, se tuvieron en cuenta los aspectos más debatidos en los últimos años sobre la enseñanza/aprendizaje a sinohablantes y se decidió la participación de dos profesores por grupo y un mediador cultural de nacionalidad china. También se tuvo en cuenta la metodología seguida actualmente en los centros chinos: el enfoque tradicional (gramática-traducción) y el estructural (Yang, 2013). Por todo ello, se abogó por una metodología ecléctica en la que se aplicaron recursos didácticos de diversa naturaleza con el fin de optimizar el aprendizaje lingüístico teniendo en cuenta las necesidades específicas de los discentes. Este enfoque permite saber si el alumno, ante una determinada dificultad de comunicación, necesita la ayuda de un experto, de los compañeros o apoyo emocional (Baralo \& Estaire, 2010).

El objetivo del estudio que aquí se presenta es el de conocer la valoración de los agentes implicados en la implantación de la metodología didáctica (uso de materiales y recursos), así como su percepción de la utilidad para el aprendizaje lingüístico. Con este fin se desarrolló un estudio observacional, descriptivo a partir de un cuestionario para cada uno de los agentes implicados: 6 profesores, 6 ayudantes y 59 alumnos. En el diseño del cuestionario se tuvieron en cuenta los factores que influyen en la enseñanza/aprendizaje mencionados anteriormente: metodología, diseño de materiales, recursos utilizados, uso de la lengua materna, reacción del alumno ante determinadas tareas, papel de profesores y ayudantes, etc. Los resultados muestran la satisfacción con la metodología, los recursos y los materiales empleados. 


\section{DIFICULTADES DE APRENDIZAJE DEL ALUMNO CHINO DE ELE}

El diseño del curso que se presenta en este trabajo y del que se muestra la valoración de los agentes implicados requiere un estudio de todos los factores que se tienen en cuenta tanto en su esbozo como en su implantación en el aula, ya sean factores lingüísticos o extralingüísticos. El objetivo del curso es desarrollar la competencia comunicativa oral de trabajadores inmigrantes chinos, por lo que hay que tener en cuenta sus dificultades y estrategias de aprendizaje, sus necesidades comunicativas, así como los aspectos más debatidos en los últimos años sobre la enseñanza/aprendizaje a sinohablantes (materiales, recursos, uso de la lengua materna, factores emocionales). Los temas más estudiados en las últimas décadas están relacionados con las dificultades mostradas por estos hablantes ante el aprendizaje del español. Una de ellas y quizás la principal, es la distancia lingüística entre las dos lenguas, sin obviar la cultural, que se refleja también en las diferencias metodológicas empleadas en el aprendizaje de lenguas. En las aulas chinas se sigue una metodología basada en el método tradicional y audiolingual, mientras que la de los profesores nativos se basa en el enfoque comunicativo. Los estudiantes chinos están acostumbrados a aprender una lengua a partir de explicaciones gramaticales explícitas y actividades de memorización y repetición, basado en el planteamiento de enseñanza propuesto por Confucio, por lo que los profesores nativos encuentran dificultades al aplicar el enfoque comunicativo (Sánchez Griñán, 2008). Las dificultades no se encuentran solamente en la presentación y explicación de contenidos gramaticales, sino también al plantear actividades comunicativas, ya que los estudiantes chinos apenas participan en estas e incluso llegan a optar por permanecer en silencio. Por ello, Sánchez Griñán (2008) plantea una propuesta didáctica en la que se tengan en cuenta las dificultades de estos alumnos ante las actividades comunicativas, y las diferencias culturales y de enseñanza entre Occidente y Oriente.

Todo esto ha sido objeto de estudio en las investigaciones de los últimos años. En ellos se aboga por una metodología ecléctica en la que se incluyan elementos tradicionales en la metodología comunicativa y se utilice la lengua china o una lengua compartida entre profesores y alumnos (Sánchez Griñán, 2008; Trapero Sandoval, 2012). Muchos profesores están integrando ya en sus clases ambas metodologías, aunque, según Yang (2013), la enseñanza mediante esta metodología tradicional se sigue manteniendo. Sin embargo, Blanco Pena (2011) habla ya de la integración de ambas metodologías, tradicional y moderna: explicaciones gramaticales, utilización de la lengua china y del inglés como lengua compartida con el docente, traducción (enunciados, instrucciones, explicaciones, etc.), inclusión de referentes culturales, secuenciación didáctica del léxico, ejercicios de traducción, práctica de repetición y memorización de manera descontextualizada, actividades comunicativas, etc. 
La escasa participación de los estudiantes en este último tipo de actividades, optando en ocasiones incluso por mantenerse en silencio, ha sido objeto de numerosos estudios en los últimos años, ya que es motivo de disgusto de gran parte de profesores nativos y chinos, que quieren implementar el enfoque comunicativo en las aulas chinas. Wang (2011) analiza el silencio al aplicar el enfoque por tareas y menciona que las correcciones del error no deben dañar la imagen del alumno para evitar la ansiedad y el silencio en el aula. Yang (2013), por su parte, recomienda el uso de manuales que presenten una visión normativa y progresiva de la gramática y no aquellos orientados a la acción, puesto que el alumno chino necesita pensar lo que va a decir antes de comenzar a hablar. Esto también se debe a las bases culturales confucionistas, ya que cometer errores hace que el estudiante pueda perder su imagen y esto le haría quedar mal ante sus compañeros. No obstante, los manuales orientados a la acción son los que ayudan al estudiante a desenvolverse en situaciones comunicativas reales. En el diseño del presente proyecto se ha tenido en cuenta todo esto, así como la motivación de los alumnos, el nivel de dominio del español en las cuatro destrezas y el conocimiento de otras lenguas, tal y como recomienda Naranjo Batidas (2010) en su estudio de análisis de necesidades de aprendizaje de ELE para sinohablantes.

\section{ESTRATEGIAS DE APRENDIZAJE DEL ALUMNADO CHINO}

La mayor parte de los estudios de estrategias de aprendizaje lingüístico de alumnos chinos se ha llevado a cabo con universitarios, por lo que contamos con escasa investigación empírica acerca de las estrategias que aplica el colectivo inmigrante en su aprendizaje y uso de la lengua en contexto de inmersión y fuera del ámbito académico.

A la hora de hablar de las estrategias de aprendizaje de estos alumnos es frecuente encontrar aserciones referentes tanto a estrategias, como a estilos y preferencias de aprendizaje, principalmente relativas a la influencia del confucianismo, la inclinación por las técnicas mnemotécnicas y la repetición, la reticencia a expresarse en público o preguntar al profesor, y el rechazo a las actividades de corte comunicativo habituales en los currículos occidentales de lengua extranjera.

No obstante, si revisamos los resultados de los últimos trabajos, podemos encontrar evidencias que cuestionan estas creencias de los docentes. Así, Pérez Villafañe (2009) concluyó que los alumnos chinos de ELE no mostraban rechazo a las formas de enseñanza basadas en la interacción oral, como tampoco al trabajo en grupo, la negociación sobre la metodología y los contenidos de enseñanza o el entrenamiento en estrategias de trabajo autónomo; más allá, manifestaban satisfacción por su efectividad. Por otra parte, también sorprende que tanto en los estudios realizados en inglés como lengua extranjera, como en los de ELE, las estrategias de memorización 
ocupen un lugar último o muy relegado respecto a otras categorías (Li, 2005; Sánchez Griñán, 2009).

Las conclusiones de Pérez Villafañe (2009) coinciden con las de estudios precedentes, lo que la autora atribuye a la adopción, fundamental, de un "enfoque ecológico y socioculturalmente sensible" (p. 12). Esta pauta se tendrá en cuenta en nuestra investigación, tanto en la formación dada a los profesores del curso Comunícate en español, como en la introducción de la figura del mediador intercultural como apoyo del docente en la elaboración de materiales y la impartición de las clases. Con ello coincidimos con Pérez Villafañe (2009) en que "las dificultades del estudiante chino para la adaptación a entornos académicos occidentales [...] son fácilmente superables siempre que se preste al estudiante un apoyo básico en los estadios iniciales" (p. 8). Igualmente, se seguirán otras dos recomendaciones de esta autora, a saber, el recurso a la retroalimentación y al entendimiento intercultural como una forma eficaz de adaptar la enseñanza al contexto educativo, y la atención a la competencia pragmática y al entrenamiento de las estrategias sociales de los alumnos.

Otros autores se sitúan en la misma línea de apostar por el acercamiento intercultural entre docente y aprendices, de considerar la influencia del contexto de enseñanza sobre las estrategias de aprendizaje, y de hacer conscientes a los alumnos de las aportaciones de las estrategias a su proceso de aprendizaje y su autonomía como usuarios de la lengua. Así, Sánchez Griñán $(2009$, p. 28) hace un análisis de la supuesta cultura china de aprendizaje en relación con las estrategias de los alumnos, para concluir que, de existir tal cultura, esta no puede sostenerse como un conjunto de elementos culturales directamente contrarios a la autonomía del aprendizaje. De acuerdo con este autor, los factores que explican la actitud, el comportamiento y las expectativas de los alumnos chinos no serían tanto las denominadas "características del aprendiente chino", como las condiciones propias de cada currículo en concreto. Por lo tanto, Sánchez Griñán (2009, p. 5) propone una superación de los estereotipos para llegar a "una sinergia cultural" en la que se produzca una toma de conciencia de las diferencias entre las culturas de aprendizaje de manera explícita, para permitir una articulación de las expectativas de docentes y aprendices, tal como postulaban Cortazzi \& Jin (1996).

Por otra parte, el autor considera responsabilidad del docente el desarrollo de la competencia estratégica del alumno, especialmente del sinohablante, como también lo hace $\mathrm{Li}$ (2005), quien incide en que debemos recordar a los estudiantes que cada contexto requiere sus estrategias y recomienda que los docentes las enseñen explícitamente y creen oportunidades para practicarlas primero en el aula, puesto que estos alumnos necesitan que se les haga conscientes del papel que juegan las estrategias en el aprendizaje lingüístico. Estas pautas se derivan de los hallazgos de Li de que los alumnos menos competentes en destrezas orales empleaban menos estrategias sociales, mientras que los de alcance léxico más limitado no reportaban usar estrategias de memorización. Independientemente de la dirección de la causalidad, dice el autor, 
se hace preciso ayudar a los estudiantes a emplear nuevas estrategias y a que hagan un uso real de la nueva lengua más allá incluso de los límites del aula.

Finalmente, los estudios anteriormente citados ponen de manifiesto una diferencia en el reporte de la frecuencia de uso y tipología de las estrategias de aprendizaje lingüístico utilizadas por los estudiantes chinos según el contexto de aprendizaje. Si esto es así comparando contextos dentro de un mismo ámbito (el académico), podemos suponer que en el caso de los inmigrantes chinos residentes en España también habrá una variación si comparamos con los resultados de las investigaciones precedentes, pues, de acuerdo con García Mateos (2008), "los inmigrantes adultos aportan un bagaje cultural, lingüístico y un savoire-faire que difiere del de los «estudiantes estándar» de L2" (p. 39).

\section{NECESIDADES COMUNICATIVAS DE LOS TRABAJADORES INMIGRANTES CHINOS}

De acuerdo con Martín Peris (2000), tomar como referencia las necesidades de los alumnos consiste en conocer el perfil del alumno respecto a las siguientes variables: el estilo de aprendizaje, las expectativas y creencias, las experiencias previas y hábitos de aprendizaje, y los factores afectivos.

En el caso que nos ocupa, las necesidades se refieren tanto a estos aspectos intrínsecos al aprendizaje, como a los relacionados con el entorno en el que deben hacer uso de la lengua meta. Para recabar estas últimas, se llevó a cabo un estudio etnográfico de observación no participativa (Porras Alba, 2015), para identificar las necesidades comunicativas de los inmigrantes adultos chinos residentes en la Comunidad de Madrid a la hora de realizar los trámites administrativos requeridos para solicitar el permiso de arraigo social y vivienda. Con este propósito, se observaron las entrevistas de la Consejería de Asuntos Sociales realizadas a inmigrantes chinos en varios centros de la Comunidad de Madrid durante un mes.

En las conclusiones de Porras Alba (2015) se manifiesta la necesidad de diseñar un curso de nivel inicial en el que los alumnos aprendan a desenvolverse en situaciones reales de la vida administrativa y pública española. Igualmente se señalan los elementos más importantes que se deben tener en cuenta respecto al comportamiento en el aula y a las dificultades de los alumnos en su competencia oral: principales errores, importancia de la cortesía china y el miedo a la pérdida del honor por verse expuesto ante situaciones que no domina, el enfrentamiento intercultural, las diferencias de jerarquía comunicativa oriental y occidental. Respecto a los contenidos, habría que seleccionar aquellos más necesarios para resolver las situaciones comunicativas a las que deben hacer frente en su día a día en la sociedad de acogida, así como las aptitudes comunicativas que los trabajadores chinos han de demostrar en las entrevistas requeridas para los trámites burocráticos de inserción social. En el 
diseño de las unidades didácticas, convendría incluir ejercicios de memorización y repetición para el aprendizaje de las estructuras lingüísticas y del léxico. Además, se debería incluir la lengua materna tanto para traducir el léxico como para facilitar el entendimiento entre profesores y alumnos.

En el proyecto Comunícate en español, se tienen en cuenta los resultados de este análisis de necesidades para el diseño y la implementación del curso y los materiales, así como para la formación de los profesores y colaboradores. En el presente trabajo, además, se mostrarán resultados de los cuestionarios de satisfacción de los alumnos, profesores y colaboradores con la metodología didáctica empleada.

\section{EL ECLECTICISMO EN EL DISEÑOS DE CURSOS}

Los últimos estudios sobre la enseñanza/aprendizaje a sinohablantes apuestan, como hemos visto anteriormente, por una metodología ecléctica en la que se combine la metodología tradicional con una más moderna. En el Marco común europeo de referencia para lenguas: aprendizaje, enseñanza, evaluación, se plantea un enfoque orientado a la acción, en tanto que los usuarios de la lengua tienen que realizar determinadas tareas en situaciones concretas y el uso de la lengua varía según el contexto. Para ello se tiene en cuenta que en el aprendizaje de lenguas hay que atender tanto a las competencias lingüísticas como a las competencias generales. Estos postulados conducen, por tanto, a una metodología ecléctica, metodología que, según Baralo \& Estaire (2010), "permite decidir con certeza si lo que necesita un alumno, ante una dificultad de comunicación determinada, es el apoyo del experto o de los compañeros para resolver una tarea, o un apoyo emocional a su autoestima" (p. 222).

En el contexto de enseñanza a trabajadores chinos seguimos una metodología ecléctica para ayudarles a desenvolverse con facilidad en determinadas situaciones comunicativas reales, ya que la metodología tradicional no favorece que los discentes sepan desenvolverse en este tipo de situaciones. Por ello, Martín Peris (en Ezeiza Ramos, 2013) apuesta por los enfoques postcomunicativos, ya que recogen todos estos aspectos integrando diferentes aportaciones conceptuales antes que excluyendo, lo que supondría una reconducción de la relación entre teóricos de la didáctica y profesores, tal como describe el autor. Además, estos enfoques no solo basan la enseñanza/aprendizaje en sus componentes lingüísticos, como ya hemos mencionado, sino también en otros factores extralingüísticos que influyen y afectan en el aprendizaje de una lengua. Ezeiza Ramos (2013) considera que habría que integrar elementos extralingüísticos inherentes a la comunicación, al uso y aprendizaje de la lengua, como la identidad lingüística, la consideración social de las lenguas, la cultura o las tradiciones educativas, pues ello conduciría a nuevos planteamientos de objetivos de aprendizaje con el fin de establecer los aspectos lingüísticos y extralingüísticos del diseño curricular. Por ello coincidimos con Baralo \& Estaire (2010) 
en que no hay una metodología ideal para todos los alumnos y que estos enfoques pueden proporcionar soluciones metodológicas apropiadas a las distintas necesidades de aprendizaje.

Los elementos lingüísticos y extralingüísticos se han tenido en cuenta en el diseño del curso atendiendo especialmente a la formación de los docentes, con el fin de no caer en un eclecticismo metodológico indiscriminado, riesgo que se corre de no formar adecuadamente al profesorado (Baralo \& Estaire, 2010). También se ha tenido en cuenta el sentido práctico fundamentado; en este sentido, Kumaravadivelu (1994) destaca de la situación posmétodo que "puede proporcionar al profesorado el conocimiento, las destrezas, la actitud y al autonomía necesarios para trazar una alternativa al método sistemática, relevante y coherente, basada en el sentido práctico fundamentado". Por ello, defiende un marco abierto y coherente basado en los actuales planteamientos teóricos, empíricos y pedagógicos que active y desarrolle el sentido de la plausibilidad de los profesores así como crearles un sentido de implicación interesada.

En cuanto a las actividades planteadas, se han combinado e integrado actividades comunicativas y de aprendizaje con el fin de que el discente se sienta familiarizado con la metodología al mismo tiempo que realiza actividades que luego le van a facilitar la comunicación con nativos en situaciones reales ${ }^{1}$ (Blanco Pena, 2011; Trapero Sandoval, 2012; Ezeiza Ramos, 2013). También es importante el uso de las nuevas tecnologías, pues permiten tanto nuevas maneras de comunicación, como el acceso a una mayor variedad de uso de la lengua (Ezeiza Ramos, 2013).

Todo esto da lugar a cursos con fines específicos atendiendo a las necesidades de los alumnos, como el programa que nos ocupa, cuyo objetivo es que los trabajadores inmigrantes chinos sean capaces de desenvolverse en situaciones del ámbito administrativo y público en la Comunidad de Madrid. En resumen, seguimos la recomendación de Baralo \& Estaire (2010) de orientar la enseñanza de la lengua hacia la acción, entendida como la capacitación de los alumnos para "hacer cosas a través de la lengua", así como "aprender en el uso y para el uso" (p. 220).

\section{LA MEDIACIÓN LINGÜÍSTICA E INTERCULTURAL EN EL APRENDIZAJE DE ELE}

La mediación lingüística se define como la traducción, interpretación, parafraseo, explicación, resumen, etc. entre interlocutores de lenguas distintas o de una misma, hablando en una lengua diferente a la propia; es decir, el mediador no expresa sus ideas sino que transmite las de otro interlocutor que no puede comunicarse

\footnotetext{
${ }^{1}$ En el caso de los trabajadores chinos, contextos administrativos para solicitar el permiso de arraigo social y vivienda.
} 
directamente, por lo que no son exclusivas de la enseñanza de lenguas. De hecho, Cassany (1996) define la figura del mediador como un profesional experto en lingüística al que recurren cada vez con mayor frecuencia agentes sociales para resolver sus necesidades lingüísticas. Por ello, las funciones que desempeña este profesional son muy variadas: traducir un informe de auditoría, interpretar una conferencia, diseñar un impreso de solicitud, corregir una carta comercial, una memoria, un comunicado de prensa, etc. En el caso que nos ocupa, la enseñanza de español a inmigrantes para facilitar su integración en la sociedad de acogida, el mediador intercultural se concibe en los términos descritos por Prats San Román \& Bermúdez Anderson (2008), como

un recurso práctico y cercano en la construcción de una competencia intercultural, aportando esos puentes en la comunicación y la relación para que puedan efectuarse en cada parte los procesos [de construcción de la competencia intercultural] en la descentración, la aproximación al otro y la negociación intercultural. (p. 74)

En el caso de la enseñanza a un colectivo que mantiene una gran distancia lingüística y cultural respecto a la lengua y cultura de acogida, la figura del mediador lingüístico se hace imprescindible al ser quien facilita la comprensión lingüística y cultural entre alumnos y profesores en las clases de ELE. La importancia del mediador lingüístico se corrobora en los resultados del estudio realizado por Barbado Gómez \& Guerra Infante (2014), en el que mostraban la necesidad de incluir la mediación lingüística e intercultural en el proceso de adquisición. En ese trabajo se empleó como recurso didáctico para resolver las dificultades que los estudiantes chinos tenían al interactuar o expresarse en español. En sus conclusiones aclaran que los profesores también ejercen como mediadores en el aula al parafrasear, apostillar, resumir, etc., pero destacan el papel de los mediadores ofrece un mayor respaldo a los aprendices y redunda en una mejora del aprendizaje lingüístico.

Para realizar su función, el mediador lingüístico emplea la lengua materna, un recurso controvertido en la enseñanza de segundas lenguas en las últimas décadas, aunque recomendado por algunos estudios dentro de unos límites y con fines pedagógicos, sin convertirla en lengua dominante (Galindo, 2011). Respecto a nuestro público meta, los investigadores aconsejan el uso de la lengua materna o de una lengua común (Blanco Pena, 2011; Trapero Sandoval, 2012).

Aparte de la mediación lingüística, en nuestro proyecto los mediadores cumplen una función clave para la integración intercultural, imprescindible en un proyecto social de estas características. Tal como describen Prats San Román \& Bermúdez Anderson (2008), despiertan el interés por el otro, orientan en el acceso a su versión de la realidad y construyen las vías de intercambio intercultural ${ }^{2}$.

\footnotetext{
2 "Las pasarelas necesarias para que podamos sumergirnos en el mundo del otro, descubrir su universo e interiorizar sus códigos de referencia” (Prats San Román \& Bermúdez Anderson, 2008, p. 74).
} 


\section{ESTUDIO EMPÍRICO}

A partir de los fundamentos de Baralo \& Estaire (2010) y Sánchez Griñán (2008), para nuestro proyecto se optó por el diseño de una metodología ecléctica en la que se combinaron elementos de la enseñanza tradicional, como son el uso de la traducción de las instrucciones y de los listados de vocabulario a la lengua materna de los alumnos, la memorización del léxico o la imitación de patrones y diálogos, con un enfoque más comunicativo. Para la implementación del curso Comunícate, cada grupo ha contado con dos profesores nativos de ELE y dos ayudantes/mediadores chinos con un nivel mínimo de español de B2. Al tratarse de un curso diseñado para alumnos de nivel inicial, el uso de la traducción al chino por parte de los mediadores se convierte en un apoyo básico para el seguimiento de la clase por parte de los aprendices. Asimismo, la práctica de memorización y recitado está relacionada, sobre todo, con la automatización de las formas, dada la enorme distancia interlingüística ( $\mathrm{Lu}, 2008)$.

Los resultados del presente trabajo muestran la valoración de los agentes implicados sobre la implementación de esta metodología didáctica, diseñada para un curso de español inicial que pretende el desarrollo de las destrezas orales de la población inmigrante china residente en la Comunidad de Madrid. Los datos de este estudio observacional y descriptivo se han recogido a través de diferentes cuestionarios administrados a tres grupos, cumplimentados por un total de 59 alumnos, 6 profesores nativos de ELE y 6 ayudantes/mediadores chinos. Los resultados que se muestran a continuación ofrecen información sobre lo siguiente:

- el grado de satisfacción de los estudiantes con respecto al método de enseñanza, sus opiniones acerca de la utilidad de tener dos profesores nativos en clase, la ayuda ofrecida por los mediadores, y los materiales y recursos utilizados en el aula;

- la opinión de los profesores nativos en cuanto al apoyo ofrecido por los ayudantes/mediadores chinos en el proceso de enseñanza;

- la valoración de los mediadores chinos en cuanto a su propio papel en la clase de ELE.

Los cuestionarios se han administrado en español para los profesores y en chino para los aprendices y mediadores. Se ha aplicado una escala de Likert de 5 puntos, considerando el 5 la máxima puntuación.

En primer lugar, al analizar las respuestas ofrecidas por los 59 alumnos del curso Comunicate ( 22 hombres y 37 mujeres de edades comprendidas entre 16 y 55 años), observamos que el 74,58\% (44 alumnos) valoran con la máxima puntuación el método de enseñanza empleado por los docentes, mientras que el 96\% (57 alumnos) consideran útil el hecho de tener dos profesores nativos en clase. En lo que respecta 
a los mediadores chinos, el 77,97\% (46 alumnos) opina que los ayudan mucho. En cuanto a los materiales y recursos ofrecidos en las sesiones lectivas, cerca del total de los alumnos opinan que las presentaciones de diapositivas proyectadas en el aula han sido útiles (57 alumnos) y divertidas (56 alumnos). La utilidad de los juegos realizados para el desarrollo de la expresión e interacción oral han sido valorados de manera muy positiva por el 93,22\% de los encuestados (55 alumnos); asimismo, al $91,52 \%$ (54 alumnos) les han resultado divertidos. Si observamos los resultados obtenidos cuando preguntamos sobre los listados de vocabulario con traducción al chino y apoyo visual, entregados a lo largo del curso, vemos que todos los informantes están totalmente de acuerdo en cuanto a su utilidad (máximas puntuaciones), aunque discrepan sobre su nivel de dificultad. Algo similar ocurre con las actividades empleadas para el desarrollo de la comprensión oral y la pronunciación, donde la gran mayoría destaca los beneficios para su aprendizaje frente a las dificultades que presentan. En último lugar, destacamos que el 100\% de los encuestados estaría dispuesto a realizar más cursos como este y lo recomendaría a otros trabajadores chinos que quisieran aprender español.

Por otra parte, los 6 docentes del curso han valorado de forma positiva la participación de los mediadores chinos en el proceso de enseñanza ( 5 de ellos con la máxima puntuación y 1 con puntuación 4) y agradecen su colaboración a la hora de traducir instrucciones, el apoyo para explicar algún contenido específico que resulta difícil para el aprendiente sinohablante, así como su papel de nexo entre profesores y alumnos para posibilitar un mayor intercambio cultural.

Finalmente, las respuestas ofrecidas por los 6 mediadores chinos (3 hombres y 3 mujeres de entre 21 y 25 años) nos indican que 5 de los informantes consideran positiva la idea de que los estudiantes sinohablantes trabajen con profesores nativos de español especialmente en la enseñanza/aprendizaje de las destrezas orales. Uno de los informantes opina, incluso, que para principiantes adultos sería conveniente el profesor chino en todo momento. No obstante, consideran que su figura dentro de un curso de estas características es necesaria principalmente a la hora de traducir determinadas instrucciones a la lengua materna de los estudiantes para el buen desarrollo de la clase, explicar un contenido gramatical concreto que, debido a la diferencia interlingüística, puede resultar de gran dificultad para este perfil de alumnado o para presentar otros contenidos que, por aspectos o diferencias culturales, deben tratarse de una manera específica, es decir, teniendo un buen conocimiento de ambas lenguas (español/chino). Ante la pregunta del cuestionario sobre la frecuencia con la que tenían que prestar ayuda al docente en clase, encontramos que 5 de nuestros informantes opinan que bastante o mucho, frente a uno que marca a veces en su respuesta. Asimismo, la cooperación entre profesores y mediadores ha sido activa, consistiendo fundamentalmente en el apoyo del uso de la lengua china dentro del aula. 


\section{CONCLUSIONES}

El proyecto Comunicate en español es un curso para trabajadores sinohablantes de la Comunidad de Madrid que pretende desarrollar su eficacia comunicativa oral en el nivel inicial de español. En el diseño del curso se han tenido en cuenta las necesidades comunicativas en el contexto de inmersión de la lengua meta, especialmente aquellas referidas a la solicitud del permiso de arraigo social y vivienda. También se han tenido en cuenta las dificultades de aprendizaje debidas a la distancia lingüística y cultural con los ciudadanos del país de acogida, el desconocimiento de la lengua y cultura china por parte de los profesores nativos y las preferencias de aprendizaje de los alumnos.

Por todo ello, se ha seguido una metodología de aprendizaje ecléctica, que se refleja en el diseño de la programación didáctica y de los materiales propuestos, en la formación del equipo docente así como en la composición de este.

La satisfacción de los agentes implicados en la primera implementación del curso avala el enfoque teórico subyacente y las acciones didácticas llevadas a cabo, dentro de las cuales destaca especialmente el papel del mediador lingüístico y cultural, muy activo durante las sesiones lectivas y positivamente valorado tanto por los docentes nativos como por los aprendices. En este sentido, convendría seguir investigando acerca de las aportaciones que un grupo de trabajo mixto, de docentes nativos y mediadores chinos, podría brindar al desarrollo e implementación de materiales didácticos en otros niveles de dominio de la lengua, así como llevar a cabo estudios que midan la eficacia de la combinación de diferentes tipologías de actividades empleadas en un curso de estas características (metodología ecléctica) para el desarrollo de la competencia lingüística comunicativa de los aprendices chinos en contexto de inmersión. 


\section{BIBLIOGRAFÍA}

Baralo, M \& Estaire, S. (2010). Tendencias metodológicas postcomunicativas. En: Ch. Abelló, Ch. Ehlers \& L. Quintana Hernández (Coords.), Escenarios bilingües: el contacto de lenguas en el individuo y la sociedad (pp. 105-128). Bern: Peter Lang. Disponible en http://www.nebrija. com/revista-linguistica/files/articulosPDF/11.BaraloEstaire.pdf

Barbado Gómez, P. \& Guerra Infante, M. R. (2014). La importancia de la mediación lingüística e intercultural en los procesos de adquisición de la lengua española para sino hablantes en contexto de inmersión: Un estudio de casos. En: N. N. Contreras Izquierdo, M. Anglada Escudé \& J. M. Blanco Pena (Eds.), La enseñanza de español para sinohablantes en contextos (Monográficos SinoELE, 10) (pp. 95-125), Jaén: SinoELE. Disponible en http://www. sinoele.org/images/Revista/10/Monograficos/EPES_2013/barbado_guerra_95-125.pdf

Blanco Pena, J. M. (2011). Principios metodológicos de la enseñanza de E/LE en contextos sinohablantes. Suplementos SinoELE, 5 (IV Jornadas de Formación de Profesores de Español Lengua Extranjera en China), 60-81.

Consejo de Europa (2001). Marco Común Europeo de Referencia para las lenguas: aprendizaje, enseñanza, evaluación. Madrid: Anaya.

Cortazzi, M. \& Jin, L. (1996). Cultures of Learning: Languages Classrooms in China. En: H. Coleman (Ed.), Society and the Language Classroom (pp. 169-206). Cambridge: Cambridge University Press.

Ezeiza Ramos, J. (2013). Influencias postcomunicativas en algunas propuestas para la enseñanza de E/LE. Revista Nebrija, 13. Disponible en http://www.nebrija.com/revista-linguistica/influ encias-postcomunicativas-en-algunas-propuestas-para-la-ense\%C3\%B1anza-de-e-le/

Galindo Merino, M. M. (2011). L1 en el aula de L2: ¿por qué no? ELUA. Estudios de Lingüística, 25,163-204.

García Manzorro, A. C. (2012). Análisis de errores en aprendientes chinos de español: estudio morfosintáctico. Suplementos SinoELE, 6. Disponible en http://www.sinoele.org/images/ Revista/6/Suplementos/cristinagarcia.pdf

García Mateos, C. (2008). La enseñanza de la L2 para inmigrantes adultos: necesidades, estrategias, dificultades y maneras de aprender. En: A. Ríos Rojas \& G. Ruiz Fajardo (Eds.), Didáctica del español como segunda lengua para inmigrantes (pp. 38-55). Sevilla: Universidad Internacional de Andalucía.

Kumaravadivelu, B. (1994). La situación posmétodo: estrategias emergentes y confluyentes para la enseñanza de segundas lenguas y de lenguas extranjeras (trad. M. Cánovas). Disponible en http://cvc.cervantes.es/ensenanza/biblioteca_ele/antologia_didactica/enfoque_comunicativo/ kumaravadivelu01.htm

Li, A. (2005). A Look at Chinese ESL Students' Use of Learning Strategies in Relation to Their English Language Proficiency, Gender and Perceived Language Difficulties - A Quantitative Study. En: Supporting Independent English Language Learning in the 21st Century: Proceedings of the Independent. Learning Association Conference Inaugural. Auckland: Manukau Institute of Technology. Disponible en http://www.independentlearning.org/uploads/1008 36/LI05051.pdf

Lu, J. (2008). Distancia interlingüística: partida de reflexiones metodológicas del español en el contexto chino. México y la cuenca del Pacifico, 11 (32): 43-54.

Martín Peris, E. (2000). La enseñanza centrada en el alumno. Algo más que una propuesta políticamente correcta. Frecuencia L, 13: 3-30. Disponible en http://cvc.cervantes.es/ensenanza/ biblioteca_ele/antologia_didactica/enfoque02/martin_peris08.htm. 
Naranjo Batidas, A. L. (2010). Planificación de un curso A1 para aprendices chinos. El análisis de necesidades previo y la fijación de objetivos. Memoria fin de máster en la enseñanza de ELE. Universidad Nebrija. Biblioteca virtual redELE. Disponible en http://www.mecd. gob.es/redele/Biblioteca-Virtual/2010/memoriaMaster/1-Trimestre/AnaLuciaNaranjo.html

Pérez Villafañe, E. (2009). De China a España: Estrategias de aprendizaje del alumno chino en ambos contextos y posibles aportaciones del profesorado hispano. Un breve estudio de casos. Suplementos MarcoELE, 8: 1-37. Disponible en http://marcoele.com/descargas/china/perez estudiodecasos.pdf

Porras Alba, E. (2015). Análisis de necesidades para el diseño del curso de ELE para inmigrantes chinos Comunicate. Trabajo fin de máster inédito, Universidad Nebrija.

Prats San Román, G. \& Bermúdez Anderson, K. (2008). La mediación intercultural: una nueva profesión para una sociedad diversa. En: A. Ríos Rojas \& G. Ruiz Fajardo (Eds.), Didáctica del español como segunda lengua para inmigrantes (pp. 72-79). Sevilla: Universidad Internacional de Andalucía.

Sánchez Griñán, J. A. (2008). Enseñanza y aprendizaje de español como lengua extranjera en China. Retos y posibilidades del enfoque comunicativo (Tesis doctoral). Murcia: Universidad de Murcia. Disponible en http://hdl.handle.net/10803/10944.

Sánchez Griñán, A. (2009). Estrategias de aprendizaje de alumnos chinos de español. Suplementos MarcoELE, 8: 1-3). Disponible en http://marcoele.com/descargas/china/sanchez-grinan_estra tegias.pdf

Trapero Sandoval, J. (2012). Las instrucciones del profesor en la enseñanza de ELE para alumnos taiwaneses de nivel inicial: incidencia de las diferencias culturales y propuesta de mejora (Trabajo fin de máster inédito). Madrid: Universidad Nebrija.

Wang, C. (2011). El análisis del silencio de los aprendices chinos de ELE y aplicación de una propuesta didáctica de expresión oral (Trabajo fin de máster inédito). Madrid: Universidad Nebrija.

Yang, T. (2013). Metodología y manuales en la enseñanza de español a sinohablantes. Revista SinoELE, 8: 17-41. Disponible en http://sinoele.org/images/Revista/8/Articulos/tyang_1741.pdf 